\title{
High inhaled corticosteroids adherence in childhood asthma: the role of medication beliefs
}

\author{
Ted Klok*, Adrian A. Kaptein\#, Eric J. Duiverman` and Paul L. Brand*
}

ABSTRACT: Our aim was to study determinants of adherence in young asthmatic children over a 3-month period, including the role of parental illness and medication perceptions as determinants of adherence.

Consecutive 2-6-yr-old children with asthma, using inhaled corticosteroids (ICS), followed-up at our paediatric asthma clinic (where patients are being extensively trained in self-management, and are followed-up closely) were enrolled. Adherence was measured electronically using a Smartinhaler ${ }_{\mathbb{B}}$ and calculated as a percentage of the prescribed dose. We examined the association of adherence to a range of putative determinants, including clinical characteristics and parental perceptions about illness and medication.

Median (interquartile range) adherence, measured over 3 months in 93 children, was 92 (7697)\%, and most children had well controlled asthma. $94 \%$ of parents expressed the view that giving ICS to their child would protect him/her from becoming worse. Adherence was significantly associated with asthma control and with parental perceptions about medication.

The high adherence rate observed in our study was associated with parental perceptions about ICS need. The high perceived need of ICS may probably be ascribed to the organisation of asthma care (with repeated tailored education and close follow-up).

KEYWORDS: Compliance, illness perceptions, medication perceptions, self-management education

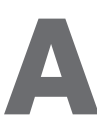
dherence to maintenance therapy is of key importance in determining the success of treatment of chronic diseases, such as childhood asthma [1,2]. Adherence to maintenance treatment, however, is poor, and this is a major cause of uncontrolled asthma [1, 3, 4]. Improving adherence to inhaled corticosteroids (ICS) in children with asthma is probably the most effective method through which healthcare providers can reduce the burden of uncontrolled asthma [1]. In contrast with the common belief among healthcare providers, parental asthma knowledge is hardly associated with adherence, and isolated educational efforts to improve asthma knowledge are ineffective in improving adherence $[1,5]$. Sociodemographic factors and the severity of asthma are also of little importance in determining adherence to maintenance treatment in this disorder [1]. Conversely, although adherence to ICS is notoriously poor among the urban-ethnic minority youth in the USA, with adherence rates between $37 \%$ and $50 \%$, high adherence can be achieved, even in such underprivileged populations, when patients are repeatedly educated about self-managing their chronic disorder and followed up closely [6, 7]. This suggests that it is not the education per se, but rather the intensity, quality and frequency of education about self-management and follow-up that help to improve adherence. Studies aimed at interventions to improve physician-patient communication have shown better adherence and asthma control in children and adults with asthma $[8,9]$.

The association between such interventions and adherence may be partially mediated by parental and patient illness and medication perceptions. Patients (and parents of paediatric patients) create their own personal cognitive representations of their illness, including beliefs about what may have caused the illness, the consequences the illness will have on their lives, how long the illness will last and whether or not it is controllable or

\section{AFFILIATIONS}

*Princess Amalia Children's Clinic, Isala Klinieken, Zwolle, \#Unit of Psychology, Leiden University Medical Centre, Leiden, and

'Beatrix Children's Hospital, University Medical Centre Groningen, Groningen, The Netherlands.

CORRESPONDENCE

T. Klok

Dr van Heesweg 2

$8025 \mathrm{AB}$

Zwolle

The Netherlands

E-mail: t.klok@isala.nl

Received:

Nov 032011

Accepted after revision: Feb 012012 First published online: Feb 232012 
curable. The cognitive and emotional representations of symptoms and illnesses are called illness perceptions [10]. Similarly, medication perceptions comprise the patient's (or parent's) cognitive and emotional representations of the medication prescribed; including method of action, desired effects and side-effects [11]. Studies in adults have shown that perceptions about illness and medication are important drivers of adherence. However, in childhood asthma care, the association between illness/medication perceptions and adherence has only been examined in qualitative or cross-sectional studies, and the importance of such perceptions as determinants of ICS adherence has not been studied in a quantitative fashion. Although asthma is very common in preschool children, only a few studies have examined adherence to maintenance therapy and its determinants in this age group [1, 11-15]. Most of these studies did not focus exclusively on young children, only two used electronic adherence monitoring, and parental illness and medication perceptions received little attention. We designed this study to assess adherence, measured electronically, and its determinants, in children aged 2-6 yrs with asthma during close follow-up in a paediatric asthma outpatient clinic. We hypothesised that adherence to ICS maintenance treatment in these patients would be associated with parental perceptions about illness and medication.

\section{METHODS}

For this study the parents of all children aged 2-6 yrs with asthma, and currently treated with ICS, attending the Princess Amalia Children's clinic (Zwolle, the Netherlands) for regular follow-up, were asked to participate in the study. All children had a doctor's diagnosis of asthma based on more than three recurrent episodes of wheezing and dyspnoea, and all were referred to our clinic by their general practitioner because of troublesome, severe or frequent symptoms. Details on the setting of the clinic and the characteristics of our comprehensive asthma care are presented in the online supplementary material. In brief, we prescribe ICS to children with asthma as daily controller therapy, in accordance with the Dutch national guidelines on paediatric asthma, which are adapted from the Global Initiative for Asthma guidelines [2]. Education and follow-up focuses on building and maintaining a strong partnership with patients and parents. We provide repeated tailored asthma self-management education, discuss parents' perceptions about asthma and its treatment, ensure concordance of treatment (goals) with parents, train correct inhalation techniques and stress the importance of adherence to daily ICS treatment [16]. To achieve this, patients and their parents visited the clinic four to six times during their first year of follow-up, and two to four times per year afterwards.

Exclusion criteria were limited knowledge of the Dutch language and severe comorbidity. We collected clinical and demographic data by structured interview and chart review. Lung function was assessed before and after inhaling salbutamol $400 \mu \mathrm{g}$; flow-volume curves were used in children $\geqslant 5$ yrs of age, and resistance of the respiratory system measured by the interrupter technique was used for children $<5$ yrs of age (Microrint ${ }^{\circledR}$; Micro Medical Ltd, Rochester, UK) [17], according to European Respiratory Society/American Thoracic Society guidelines. Results were expressed as z-scores.
Upon entry into the study, a number of validated questionnaires were applied; details including references are presented in the online supplementary material. Parental illness perceptions were assessed by the Brief Illness Perception Questionnaire, and medication perceptions by the Beliefs about Medicines Questionnaire (BMQ), which also provides the balance between parentperceived necessity and concerns about ICS, and the Treatment Satisfaction Questionnaire for Medication (TSQM) [11, 18, 19]. In addition, we applied the I Worry scale, which scored parental worries about their child having asthma and using daily ICS; the Medication Adherence Report Scale (MARS), which assessed self-reported adherence; the Satisfaction with Information about Medicines Scale; the Paediatric Asthma Caregiver Quality of Life questionnaire; and an asthma knowledge questionnaire.

Asthma control was assessed by a parent-completed Asthma Control Questionnaire [20]. The attending physician rated asthma control on a visual analogue scale ranging from 0 (worst asthma control possible) to 10 (complete asthma control) at a 3 month follow-up visit.

Patients used ICS by metered dose inhaler/spacer combination during the 3-month follow-up period. Adherence was monitored by Smartinhaler ${ }^{\circledR}$ (Nexus6 Ltd, Auckland, New Zealand) a validated electronic device logging date and time of each ICS actuation [21].

\section{Analysis}

Adherence was calculated as the number of Smartinhalerrecorded inhaled doses expressed as a percentage of the number of doses prescribed, and censored at $100 \%$ of the prescribed dose (see online supplementary material for further details). We assessed the association of ICS adherence (both as a continuous variable and dichotomised as good $(>80 \%)$ and poor $(\leqslant 80 \%)$ adherence of prescribed doses used) to all putative determinants (defined a priori with a focus on parental perceptions about illness and medication; see online supplementary material) in non-parametric univariate analyses (because adherence had a highly skewed distribution) [12]. We chose to refrain from adjustments for multiple comparisons because of the exploratory and observational nature of our study.

\section{Ethical considerations}

This study was approved by the hospital ethics review board and all parents provided written informed consent.

\section{RESULTS}

Out of a total of 137 consecutive eligible patients, 103 children (75\%) entered the study after informed consent was obtained, and 93 children (90\% of those enrolled) completed the 3 month follow-up (fig. 1). Most parents who did not participate in or withdrew their child from the study did so because of serious illness in a family member or other pressing circumstances. Clinical characteristics of participating and non-participating children were comparable (table 1). Most participating children had well controlled asthma while on ICS maintenance therapy. Reliable and reproducible lung function results could be recorded in $66(71 \%)$ children, and sensitisation to inhalant allergens was available for 86 children (table 1).

The frequency distribution of adherence to daily ICS is presented in figure 2. Median (interquartile range (IQR)) adherence was 


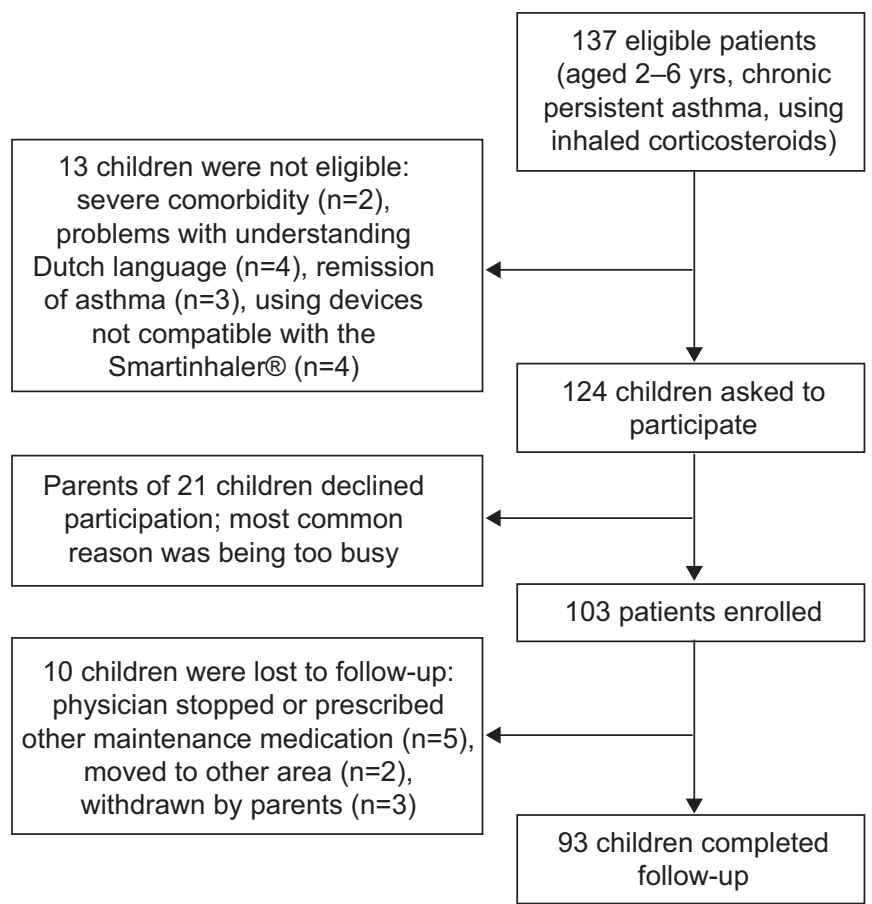

FIGURE 1. Inclusion of patients.

$92(76-97) \% .67(72 \%)$ children had good adherence $(>80 \%$ of prescribed dosages); adherence rates $<60 \%$ were recorded in only eight $(9 \%)$ children (fig. 2$)$. There was a small, non-significant decrease in adherence from the first to the third month of followup (median $(\mathrm{IQR})-1(-8-1) \%$; $\mathrm{p}=0.483)$. Adherence rates were comparable between children aged 2-4 yrs (median 92\%) and children aged 5-6 yrs (median 92\%, $\mathrm{p}=0.899$ ).

The association of adherence to the individual item responses on each questionnaire is presented in the online supplementary material. Parental perceptions about illness were not associated with adherence, but perceptions about medications were. Parents who believed that ICS maintenance treatment is needed for their child's health, those who viewed ICS administration as convenient, and those who expected little harm from medicines in general had higher adherence rates to ICS (table 2). The other putative determinants that showed significant $(p<0.05)$ or near-significant $(p<0.1)$ association to adherence are presented in table 3. Not unexpectedly, parental selfreported adherence (as assessed by MARS) was strongly related to electronically measured adherence. In addition, good asthma control was positively related to adherence measured electronically. Parental characteristics such as educational level, asthma knowledge and smoking habits were not significantly associated with adherence (see online supplementary material for details).

For $84(93 \%)$ parents, BMQ scores indicated that the perceived necessity outweighed concerns about ICS (table 4). This was mainly due to the large majority of parents expressing the view that giving ICS to their child would benefit his/her health, even when they had concerns about the use of ICS. The results from other questionnaires were consistent with this finding (see online supplementary material for details). For example, only three parents responded negatively to the TSQM question "Overall, how confident are you that taking this medication is a good thing for your child?".

\section{TABLE 1 Characteristics of all eligible study patients}

Patients with complete follow-up All other eligible patients p-value

\section{Subjects $n$}

Male

Age yrs

Duration of outpatient clinic asthma care before study months

Maintenance medication

ICS

ICS and long-acting bronchodilators

ICS (fluticasone) dose $\mu \mathrm{g}$

Hospitalisations in yr before study

ACQ baseline $(<0.75=$ well controlled, $>1.5=$ not well controlled)

PACQoL baseline (score 1-7; 1=low, 7 =high)

Smoking parent(s)

Educational level of mother ( $1=$ low, 7 =high)

Positive specific IgE to common inhalant allergens

FEV1 baseline ${ }^{\#}$

FVC baseline ${ }^{\#}$

Rrs,int baseline ${ }^{\#}$
Scheduled visits to outpatient clinic in yr before study

93
$56(61)$
$4.5(2.2-6.8)$
$17(8-27)$
$86(92)$
$7(8)$
$250(125-500)$
$5(4-6)$
$0(0-5)$
$0.5(0.2-1.3)$
$6.2(5.3-6.8)$
$28(30)$
$5(4-7)$
$43(50)$
$0.66 \pm 1.1$
$0.29 \pm 1.3$
$1.90 \pm 2.8$

$\begin{array}{cc}44 & \\ 26(60) & 0.90 \\ 4.6(2.1-6.9) & 0.67 \\ 17(9-28) & 0.58 \\ & \\ 42(95) & 0.51 \\ 2(5) & 0.51 \\ 250(125-500) & 0.73 \\ 4(2-5) & 0.007 \\ 0(0-1) & 0.07 \\ N A & N A \\ N A & N A \\ N A & N A \\ N A & N A \\ 24(65)^{+} & <0.001 \\ N A & N A \\ N A & N A \\ N A & N A\end{array}$

Data are presented as n (\%), median (interquartile range) or mean \pm SD, unless otherwise specified. ICS: inhaled corticosteroids; ACQ: Asthma Control Questionnaire [20] PACQoL: Paediatric Asthma Caregiver Quality of Life Questionnaire; Ig: immunoglobin; FEV1: forced expiratory volume in 1 s; FVC: forced vital capacity; Rrs, int: resistance of respiratory system measured by the interrupter technique; NA: not available. ${ }^{*}:$-score, $n=33 ;{ }^{\bullet}: n=86 ;^{+}: n=37$. 


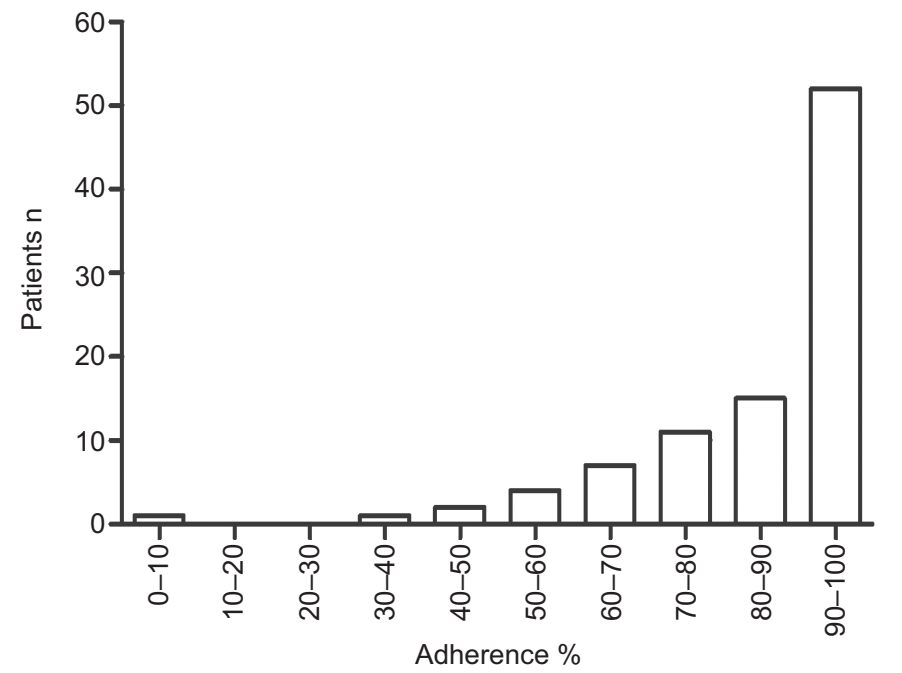

FIGURE 2. Adherence to inhaled corticosteroids measured by electronic loggers.

\section{DISCUSSION}

This study shows that high adherence to daily maintenance treatment with ICS along with good asthma control can be achieved in preschool children with asthma and that high adherence is associated with parental beliefs about the necessity of ICS therapy. The median adherence rate in our study remained stable over 3 months of follow-up at $92 \%$, which is considerably higher than in earlier studies (adherence ranging from $44 \%$ to $72 \%$, decreasing strongly over time). For example, a recent study showed that adherence to an ICS prescribed after an emergency visit for acute asthma deteriorated from $90 \%$ to $50 \%$ during the first 2 weeks after the acute asthma attack [22]. This confirmed earlier population-based observations that most patients with asthma fail to continue ICS use after an initial prescription [23]. Although some large studies showed lower adherence rates for adolescents than for school- and preschool-aged children, age is not consistently related to treatment adherence [1]. Even in pre-adolescent children, adherence to ICS of $>75 \%$ of prescribed dosages has rarely been described, neither in observational, nor in intervention studies [14]. We emphasise that the very high adherence we observed was not the result of an intervention aimed at improving adherence, but was obtained during routine care in our paediatric asthma outpatient clinic.

The large majority of parents of the preschool children with asthma in our study expressed the belief that ICS therapy for their child's asthma was both useful and necessary (table 4). This is in contrast with previous studies examining medication beliefs in asthma. Studies in adults with asthma have shown lower BMQ "need" scores and higher "concern" scores [11, 24]. In a study of 622 parents of children with asthma in the USA, concern scores exceeded need scores in $17 \%$ of parents, compared to only $4 \%$ in our study [15]. Such concerns about the daily use and safety of ICS in children are a major reason

TABLE 2 Associations between parental perceptions about illness and medication and electronically measured adherence

\begin{tabular}{|c|c|c|c|}
\hline & \multicolumn{2}{|c|}{ Rank correlation coefficient } & $\begin{array}{c}\text { Comparison between children with high }(>80 \%) \\
\text { and low adherence }(<80 \%) \text { of prescribed } \\
\text { dosages taken }\end{array}$ \\
\hline \multicolumn{4}{|l|}{ Brief IIIness Perception Questionnaire } \\
\hline Personal control & 0.11 & 0.319 & 0.760 \\
\hline Treatment control & 0.11 & 0.294 & 0.526 \\
\hline Identity & -0.05 & 0.663 & 0.598 \\
\hline Concern & 0.00 & 0.970 & 0.835 \\
\hline Understanding & -0.08 & 0.440 & 0.260 \\
\hline Emotional response & 0.13 & 0.203 & 0.961 \\
\hline Need-concern ratio & 0.09 & 0.414 & 0.394 \\
\hline General part: perceptions of overuse & 0.181 & 0.082 & 0.418 \\
\hline General part: perceptions of harm & 0.233 & $0.025^{\star}$ & 0.253 \\
\hline \multicolumn{4}{|c|}{ Treatment Satisfaction Questionnaire for Medication } \\
\hline Effectiveness of daily use of ICS & -0.10 & 0.342 & 0.633 \\
\hline Side effects of daily use of ICS & -0.01 & 0.963 & 0.838 \\
\hline Convenience of administrating ICS to child & -0.25 & $0.020^{*}$ & 0.212 \\
\hline Global satisfaction about ICS & -0.19 & 0.067 & 0.522 \\
\hline
\end{tabular}


TABLE 3 Other putative determinants which showed a (near) significant $(p<0.1)$ association with electronically measured adherence over 3-month period

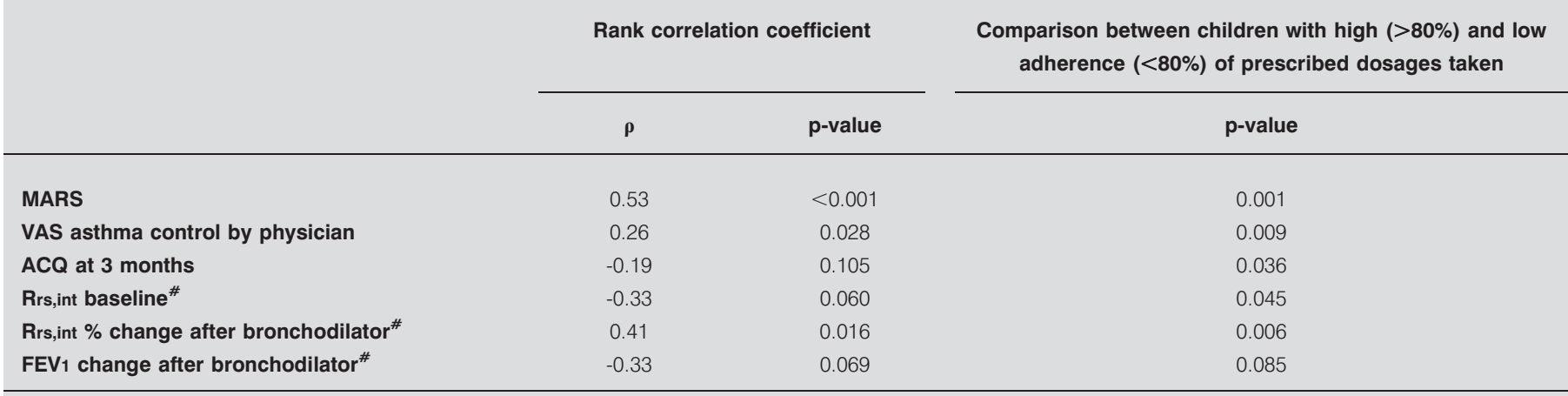

MARS: Medication Adherence Report Scale; VAS: visual analogue scale; ACQ: Asthma Control Questionnaire [20]; Rrs,int: resistance of the respiratory system measured by the interrupter technique; FEV1: forced expiratory volume in $1 \mathrm{~s}$ before and after salbutamol. ${ }^{\#}: \mathrm{n}=33$.

for parents to withdraw this medication in their children [25]. Because the distributions of ICS adherence (fig. 2) and parental medication perceptions (table 4) were strongly skewed towards high adherence and ICS necessity, with relatively little variance, the power of our study to identify determinants for adherence was lower than expected. This may help to explain why we only identified a few parental perceptions significantly associated with adherence rates, and why these associations were relatively weak (tables 2 and 3 ).

We found a significant association between ICS adherence and asthma control, with poorer asthma control in patients with lower adherence, suggesting that this association does not only play a role at the severe end of the asthma spectrum, but also in patients with relatively well controlled asthma, and that every effort to optimise adherence to ICS maintenance therapy is worthwhile.
It is interesting to explore potential reasons why the parental medication beliefs and the high adherence rates in our study population differed from those previously reported. Selective enrolment of parents with high need and low concern scores should be considered. However, we feel that this is unlikely, because consecutive outpatients at our clinic were approached for the study, the large majority of whom were enrolled (fig. 1). Furthermore, the high need scores for ICS in this study are in agreement with a previous qualitative study from our clinic, in which parents accepted that their child needed ICS to control asthma, although parents universally expressed reservations about having to give daily medication to their child. Parents whose children were treated in primary care, however, expressed beliefs of the high concern-low need pattern. These findings prompted us to hypothesise that parental medication beliefs can be modified by repeated tailored education and close follow-up in a specialised asthma clinic, resulting in high adherence [26].

TABLE 4 Parental beliefs about medication measured by the Beliefs about Medicines Questionnaire [11]

\begin{tabular}{|c|c|c|c|}
\hline \multicolumn{4}{|l|}{5 questions about need } \\
\hline My child's life would be impossible without his/her medicines & 26.7 & 35.6 & 37.8 \\
\hline Without his/her medicines my child would be very ill & 51.1 & 23.3 & 25.6 \\
\hline My child's health in the future will depend on his/her medicines & 24.7 & 43.8 & 31.5 \\
\hline My child having to take medicines worries me & 39.3 & 6.7 & 53.9 \\
\hline I sometimes worry about long-term effects of my child's medicines & 44.9 & 18.0 & 37.1 \\
\hline My child's medicines are a mystery to me & 4.5 & 7.9 & 87.6 \\
\hline My child's medicines disrupt his/her life & 1.1 & 3.3 & 95.6 \\
\hline I sometimes worry about my child becoming too dependent on his/her medicines & 28.9 & 10.0 & 61.1 \\
\hline
\end{tabular}

Data are presented as \%. 
The young age of the children in our study may have increased the likelihood of good adherence, because most medication in this age range is given by the parents. However, previous studies have shown poor adherence rates to inhaled medication in young children with asthma, even when parents knew that adherence was being monitored [14]. Therefore, parental awareness of monitoring adherence does not seem a likely cause of the high adherence rates observed. This is supported by the finding that adherence rates did not deteriorate significantly over time in our study. Further follow-up of this cohort will allow us to examine whether the high adherence rates observed can be maintained over longer periods of time.

It should also be noted that access to healthcare and ICS medication is available to all Dutch citizens, because health insurance is mandatory in the Netherlands. Studies in urban US populations have shown that poor insurance and financial issues may play a major role in poor adherence in such populations [1, 6]. Although it is conceivable that excellent insurance coverage may have improved adherence in our study, poor adherence has been shown in earlier Dutch studies of asthmatic children, suggesting that this is not a major determinant $[4,26]$.

In the absence of other logical explanations for the high adherence and constructive parental medication beliefs observed, we hypothesise that the organisation of our asthma care may be a contributing factor. The fact that our clinic is run by paediatric asthma specialists may be of importance in this respect. Previous studies have shown superior asthma control, better lung function and higher adherence to treatment and selfmanagement plans when asthma care was provided by specialist physicians (paediatric allergists or paediatric pulmonologists, depending on country and setting) compared with general paediatricians or family physicians [3, 27, 28]. A recent systematic review showed that more intensive follow-up, with multiple educational sessions using combinations of instructional modalities, was associated with higher adherence and improved outcomes for children with asthma [3]. In our clinic, such a comprehensive asthma management consists of frequent follow-up visits to both asthma specialist physicians and dedicated asthma nurses, providing repeated tailored education to parents and patients and extensively training and checking correct inhalation technique [16]. In earlier studies, we reported the added value of easy accessibility of asthma nurses which was highly appreciated by parents of children with asthma $[26,29]$. This was recently confirmed in a study from a referral centre for children with difficult-to-treat asthma, where the important role of nurse-led home visits to address parental perceptions, contextual and psychosocial issues was highlighted [30]. Taken together, these results suggest that intensive multidisciplinary education about self-management, along with close follow-up in a specialised clinic setting, helps to improve adherence to ICS in children with asthma, with parental perceptions about medication being an important mediator.

It should be stressed, however, that a causal relationship between our model of care and the high adherence we observed cannot be established based on our data because this was an observational study. Ideally, a randomised controlled trial should be performed to test the hypothesis that comprehensive asthma management and close follow-up, as described, leads to high adherence to maintenance medication. However, it has been argued that randomised trials on the effects of complex interventions, such as our model of asthma management, are fraught with difficulties [16]. A prospective study in which both adherence and parental perceptions about medicines are followed-up over longer periods of time from the time of referral to a specialised asthma clinic would also be worthwhile.

\section{Strengths and limitations}

The main strengths of our study include the objective, validated, quantitative assessment of adherence over a considerable period of time and the extensive characterisation of patients' and parents' characteristics using validated methodology. The reallife setting avoids any adherence-improving effects of clinical trial interventions, other than the potential adherence-improving effect of participating in a study. This effect is an unavoidable drawback of electronic adherence monitoring.

The most important limitation of our study lies in its generalisability. We limited our study to 3 month adherence in children aged 2-6 yrs in a specialised asthma clinic. Further follow-up of this cohort and additional studies in other settings are needed to substantiate our observation that high adherence combined with well controlled asthma is possible in other patient groups. Recent studies from other groups support the idea that more intensive asthma care enhancing patient-provider partnership improves adherence and asthma control, and decreases hospitalisation rates and asthma expenditures $[7,28]$.

\section{Conclusions}

This study shows very high 3-month adherence rates to ICS maintenance treatment in children aged 2-6 yrs with asthma. This high adherence was associated with improved asthma control, and with parental medication perceptions, which are in agreement with the chronic illness model of asthma. We hypothesise that this desirable combination of high adherence and constructive parental medication beliefs can be ascribed to the organisation and content of asthma care, with repeated tailored self-management training and close follow-up, which helps to build and maintain a strong partnership between patients/parents and the medical team [2]. Further studies are needed to examine the relationship of such guideline-driven comprehensive asthma treatment and high adherence.

\section{SUPPORT STATEMENT}

This study was supported by a grant from the Netherlands Asthma Foundation (grant no 3.4.06.007).

\section{STATEMENT OF INTEREST}

A statement for P.L. Brand can be found at www.erj.ersjournals.com/ site/misc/statements.xhtml

\section{REFERENCES}

1 Drotar D, Bonner MS. Influences on adherence to pediatric asthma treatment: a review of correlates and predictors. J Dev Behav Pediatr 2009; 30: 574-582.

2 Global Initiative for Asthma (GINA). Global Strategy for Asthma Management and Prevention. Updated 2010. Available from: www.ginasthma.org Date last accessed: September 12, 2011.

3 Bravata DM, Gienger AL, Holty JE, et al. Quality improvement strategies for children with asthma: a systematic review. Arch Pediatr Adolesc Med 2009; 163: 572-581. 
4 van Dellen QM, Stronks K, Bindels PJ, et al. Adherence to inhaled corticosteroids in children with asthma and their parents. Respir Med 2008; 102: 755-763.

5 Dean AJ, Walters J, Hall A. A systematic review of interventions to enhance medication adherence in children and adolescents with chronic illness. Arch Dis Child 2010; 95: 717-723.

6 McNally KA, Rohan J, Schluchter M, et al. Adherence to combined montelukast and fluticasone treatment in economically disadvantaged African American youth with asthma. J Asthma 2009; 46: 921-927.

7 Scott L, Morphew T, Bollinger ME, et al. Achieving and maintaining asthma control in inner-city children. J Allergy Clin Immunol 2011; 128: 56-63.

8 Wilson SR, Strub P, Buist AS, et al. Shared treatment decision making improves adherence and outcomes in poorly controlled asthma. Am J Respir Crit Care Med 2010; 181: 566-577.

9 Cabana MD, Slish KK, Evans D, et al. Impact of physician asthma care education on patient outcomes. Pediatrics 2006; 117: 2149-2157.

10 Kaptein AA, Klok T, Moss-Morris R, et al. Illness perceptions: impact on self-management and control in asthma. Curr Opin Allergy Clin Immunol 2010; 10: 194-199.

11 Horne R, Weinman J, Hankins M. The Beliefs about Medicines Questionnaire: the development and evaluation of a new method for assessing the cognitive representation of medication. Psychology and Health 1999; 14: 1-24.

12 Lasmar L, Camargos P, Champs NS, et al. Adherence rate to inhaled corticosteroids and their impact on asthma control. Allergy 2009; 64: 784-789.

13 Smith LA, Bokhour B, Hohman KH, et al. Modifiable risk factors for suboptimal control and controller medication underuse among children with asthma. Pediatrics 2008; 122: 760-769.

14 Gibson NA, Ferguson AE, Aitchison TC, et al. Compliance with inhaled asthma medication in preschool children. Thorax 1995; 50: 1274-1279.

15 Conn KM, Halterman JS, Lynch K, et al. The impact of parents' medication beliefs on asthma management. Pediatrics 2007; 120: e521-e526.

16 Brouwer AF, Brand PL. Asthma education and monitoring: what has been shown to work. Paediatr Respir Rev 2008; 9: 193-199.

17 Beydon N, M'Buila C, Bados A, et al. Interrupter resistance shortterm repeatability and bronchodilator response in preschool children. Respir Med 2007; 101: 2482-2487.
18 Broadbent E, Petrie KJ, Main J, et al. The Brief Illness Perception Questionnaire. J Psychosom Res 2006; 60: 631-637.

19 Atkinson MJ, Sinha A, Hass SL, et al. Validation of a general measure of treatment satisfaction, the Treatment Satisfaction Questionnaire for Medication (TSQM), using a national panel study of chronic disease. Health Qual Life Outcomes 2004; 2: 12.

20 Juniper EF, Bousquet J, Abetz L, et al. Identifying "wellcontrolled" and "not well-controlled" asthma using the Asthma Control Questionnaire. Respir Med 2006; 100: 616-621.

21 Burgess SW, Wilson SS, Cooper DM, et al. In vitro evaluation of an asthma dosing device: the smart-inhaler. Respir Med 2006; 100: 841-845.

22 Ducharme FM, Zemek RL, Chalut D, et al. Written action plan in pediatric emergency room improves asthma prescribing, adherence, and control. Am J Respir Crit Care Med 2011; 183: 195-203.

23 Hasford J, Uricher J, Tauscher M, et al. Persistence with asthma treatment is low in Germany especially for controller medication a population based study of 483,051 patients. Allergy 2010; 65: 347-354.

24 Menckeberg TT, Bouvy ML, Bracke M, et al. Beliefs about medicines predict refill adherence to inhaled corticosteroids. J Psychosom Res 2008; 64: 47-54.

25 Yoos HL, Kitzman H, Henderson C, et al. The impact of the parental illness representation on disease management in childhood asthma. Nurs Res 2007; 56: 167-174.

26 Klok T, Brand PL, Bomhof-Roordink H, et al. Parental illness perceptions and medication perceptions in childhood asthma, a focus group study. Acta Paediatr 2011; 100: 248-252.

27 Diette GB, Skinner EA, Nguyen TT, et al. Comparison of quality of care by specialist and generalist physicians as usual source of asthma care for children. Pediatrics 2001; 108: 432-437.

28 Kelly CS, Morrow AL, Shults J, et al. Outcomes evaluation of a comprehensive intervention program for asthmatic children enrolled in Medicaid. Pediatrics 2000; 105: 1029-1035.

29 Kamps AW, Brand PL, Kimpen JL, et al. Outpatient management of childhood asthma by paediatrician or asthma nurse: randomised controlled study with one year follow up. Thorax 2003; 58: 968-973.

30 Bracken M, Fleming L, Hall P, et al. The importance of nurse-led home visits in the assessment of children with problematic asthma. Arch Dis Child 2009; 94: 780-784. 